\title{
Article \\ Self-Organized PT-Symmetry of Exciton-Polariton Condensate in a Double-Well Potential
}

\author{
Panayotis A. Kalozoumis $1,2,3, *$ (D) and David Petrosyan ${ }^{3,4}$ (D) \\ 1 Materials Science Department, School of Natural Sciences, University of Patras, GR-26504 Patras, Greece \\ 2 Department of Informatics and Engineering, Hellenic American University, 436 Amherst st, \\ Nashua, NH 03063, USA \\ 3 Institute of Electronic Structure and Laser, FORTH, GR-70013 Heraklion, Greece; dap@iesl.forth.gr \\ 4 A. Alikhanyan National Science Laboratory (YerPhI), Yerevan 0036, Armenia \\ * Correspondence: pkalozoumis@hauniv.edu
}

Citation: Kalozoumis, P.A.; Petrosyan, D. Self-Organized PT-Symmetry of Exciton-Polariton Condensate in a Double-Well Potential. Appl. Sci. 2021, 11, 7372. https://doi.org/10.3390/app11167372

Academic Editor: Serghei Klimin

Received: 19 June 2021

Accepted: 5 August 2021

Published: 11 August 2021

Publisher's Note: MDPI stays neutral with regard to jurisdictional claims in published maps and institutional affiliations.

Copyright: (c) 2021 by the authors. Licensee MDPI, Basel, Switzerland. This article is an open access article distributed under the terms and conditions of the Creative Commons Attribution (CC BY) license (https:// creativecommons.org/licenses/by/ $4.0 /)$.
Abstract: We investigate the dynamics and stationary states of a semiconductor exciton-polariton condensate in a double-well potential. We find that upon the population build-up of the polaritons by above-threshold laser pumping, coherence relaxation due to the phase fluctuations in the polaritons drives the system into a stable fixed point corresponding to a self-organized PT-symmetric phase.

Keywords: exciton-polaritons; PT-symmetry; double-well potential; Bose-Einstein condensates

\section{Introduction}

One of the prominent research directions in semiconductor optics is the study of exciton-polariton condensation in microcavities. Exciton-polaritons are hybrid quasiparticles of strongly coupled quantum well excitons and cavity photons [1,2], retaining the properties of both matter and light. The excitonic part mediates effective interactions between the polaritons, giving rise to interesting nonlinear properties, whereas the small effective mass of the photonic component enables Bose-Einstein condensation even at ambient temperatures [3-5], in contrast to their ultracold atomic counterparts [6]. The short lifetime of the polariton condensate renders it an open system that requires continuous replenishing from the excitonic reservoir via external pumping. After their experimental realization $[7,8]$, the polariton condensates were shown to be an ideal system for studies of many effects at the interface between non-equilibrium physics and nonlinear dynamics.

The intrinsic nonlinear dynamics of polariton systems lead to a variety of effects, such as the appearance of a Mach-Cherenkov cone in a supersonic flow [9], the formation of quantized vortices [10], and dark solitons [11]. Moreover, the polariton condensates can be engineered with high precision by the external laser fields [8,12-18]. Finally, such systems are promising candidates for various applications in photonic devices, such as switches, gates and transistors [3], as well as for simulations of interacting spin models [19,20].

The "open" nature of a system featuring gain and loss leads to interesting implications when the dissipative dynamics become pseudo-Hermitian. This is the case in parity-time (PT) symmetric setups, where dissipation losses are exactly balanced by the pumping gain. Systems with PT-symmetry have been a flourishing and broad research field, extending from quantum mechanics [21] and field theory [22] to optics [23] and acoustics [24].

The interplay between the inherent losses and the laser pumping adjusted to preserve the PT symmetry provides an effective framework where a polariton system can exhibit coherent, Hermitian-like dynamics for a relatively long time. Remarkable recent results include permanent Rabi oscillations [25], multistability and condensation below the threshold [26], exceptional points in polaritonic cavities below the lasing threshold [27], dynamic phenomena at a critical exceptional point [28], and coherent oscillations of a two-species polariton mixture in a double well [29]. The latter has been shown to be able to simulate 
the dynamics of a pair of spin- $1 / 2$ particles (qubits) in the presence of exchange interaction. However, to date, polariton structures have not been extensively studied in the framework of PT symmetry, and more efforts are needed to reveal the rich landscape of phenomena that can emerge from this framework.

In this work, we study the dynamics of an exciton-polariton condensate in a double well potential, in the presence of time-varying exciton populations and phase fluctuations. We consider the coupled-mode Gross-Pitaevskii equations for the polaritons supplemented by the rate equations for the laser-pumped exciton reservoirs, and analytically derive the steady state solutions for the exciton and polariton populations, as well as their coherence. We find that, when the total pumping rate is above threshold, the system automatically attains the PT symmetric state, independently of the pumping rates of the individual sites. By employing numerical simulations for different pumping rates and initial conditions, we verify our analytical findings. We also study the stability and robustness of our results in the presence of phase noise caused by the unavoidable phase fluctuations in the polaritons.

\section{The Exciton-Polariton System}

The system under consideration is schematically illustrated in Figure 1. One or more layers of semiconductor quantum wells are placed inside the semiconductor microcavity near the antinode of the resonant cavity field mode. Spatially shaped pumping lasers replenish continuously the exciton reservoirs and simultaneously create confining potentials for the polariton condensate. Assuming a tight-binding double-well potential, the exciton-polariton system can be described by the following coupled non-linear Schrödinger equations for the polariton condensate wavefunctions $\psi_{L}$ and $\psi_{R}$ in the left $(L)$ and right $(R)$ wells [30]:

$$
\begin{aligned}
i \partial_{t} \psi_{L} & =\left[\epsilon_{L}+\eta\left|\psi_{L}\right|^{2}\right] \psi_{L}+\frac{i}{2}\left[R n_{L}-\kappa\right] \psi_{L}-J \psi_{R}, \\
i \partial_{t} \psi_{R} & =\left[\epsilon_{R}+\eta\left|\psi_{R}\right|^{2}\right] \psi_{R}+\frac{i}{2}\left[R n_{R}-\kappa\right] \psi_{R}-J^{*} \psi_{L},
\end{aligned}
$$

where $\epsilon_{L, R}$ are the single-particle energies, $\eta$ is the nonlinear interaction strength, $\kappa$ is the decay rate of the polaritons due to the exciton recombination and cavity photon losses (assumed the same for both wells), and $J$ is the Josephson (tunnel) coupling between the wells. The polariton equations are supplemented by the equations for the populations $n_{L, R}$ of the reservoir excitons,

$$
\begin{aligned}
& \partial_{t} n_{L}=P_{L}-\Gamma n_{L}-R n_{L}\left|\psi_{L}\right|^{2}, \\
& \partial_{t} n_{R}=P_{R}-\Gamma n_{R}-R n_{R}\left|\psi_{R}\right|^{2},
\end{aligned}
$$

which are created by laser pumping with rates $P_{L, R}$, decay with rate $\Gamma$, and scatter into the polariton condensate with rate $R$.
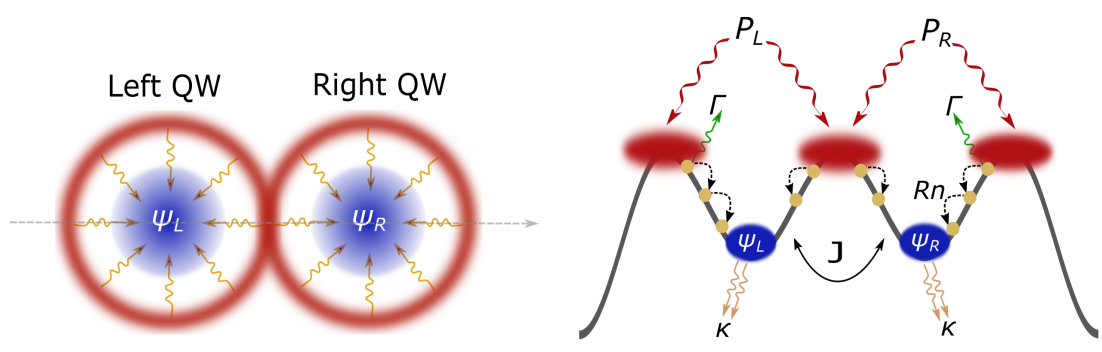

Figure 1. Schematic top view (left panel) and side energy view (right panel) of a polariton system in a double quantum well. Spatially shaped pumping lasers populate with rates $P_{L}$ and $P_{R}$ the reservoir excitons $n_{L, R}$, which decay via recombination with rates $\Gamma$ and energy-relax and scatter into the polariton condensate with rate $R$. The pumping lasers also create the confining potentials for the polaritons $\psi_{L}$ and $\psi_{R}$, which decay with rates $\kappa$, and are continuously replenished by reservoir excitons with rates $R n_{L, R}$, while interacting with each other via the Josephson coupling $J$. 
In Appendix A, we briefly outline the PT-symmetry conditions for a condensate in a double well potential. Momentarily neglecting the non-linearity $\eta$, the PT-symmetry condition is satisfied when $\epsilon_{L, R}=\epsilon(=0)$ and the gain in one well exactly compensates the losses in the other,

$$
\gamma_{L} \equiv \frac{1}{2}\left[R n_{L}-\kappa\right]=-\frac{1}{2}\left[R n_{R}-\kappa\right] \equiv-\gamma_{R},
$$

as per Equations (1a) and (1b), which leads to $n_{L}+n_{R}=\frac{2 \kappa}{R}$. The threshold pumping at which the polariton condensate starts to form can be obtained from the condition that the sum of the gain and loss in both wells is non-negative, $\gamma_{L}+\gamma_{R} \geq 0$. With Equation (3), this condition is equivalent to

$$
n_{L}+n_{R} \geq \frac{2 \kappa}{R}
$$

Note that, exactly at the threshold, this is the same condition as for the PT-symmetry. In the stationary regime for the reservoir excitons, $\partial_{t} n_{L, R}=0$, we find from Equation (2) the steady-state values

$$
n_{L, R}=\frac{P_{L, R}}{\Gamma+R\left|\psi_{L, R}\right|^{2}}
$$

Exactly at the threshold for condensate formation, the values of the polariton populations in both wells, $\left|\psi_{L, R}\right|^{2}$, are marginally equal to zero, and we have $n_{L, R} \simeq P_{L, R} / \Gamma$. Substituting these values into Equation (4), we find the threshold pumping condition

$$
P_{L}+P_{R} \geq \frac{2 \kappa \Gamma}{R}
$$

above which the condensate begins to form, while for $P_{L}+P_{R}<2 \kappa \Gamma / R$ the condensate decays to zero.

In the upper panels of Figure 2, we show the polariton populations $\left|\psi_{L, R}\right|^{2}$ for different pumping rates and initial conditions, with and without non-linear interaction, as obtained from the numerical solution to Equations (1) and (2). The insets show the evolution of the exciton populations $n_{L}$ and $n_{R}$ and their sum $n_{L}+n_{R}$. For pumping below threshold, we observe a decay in the initial (seed) polariton populations with rate $\gamma_{L}+\gamma_{R} \simeq \frac{R}{2 \Gamma}\left(P_{L}+P_{R}\right)-\kappa<0$, accompanied by Rabi-like oscillations, while the exciton populations settle to $n_{L, R}=P_{L, R} / \Gamma$. For pumping above threshold, the polariton populations grow until they reach certain values $\left|\psi_{L, R}\right|^{2}$ at which $n_{L}+n_{R} \simeq \frac{2 \kappa}{R}$, while the Rabi-like oscillations persist or are eventually damped, depending on the initial conditions or presence of non-linear interaction, as discussed below. Remarkably, the polariton and exciton populations increase and decrease, respectively, reaching the same stationary values which satisfy the PT-symmetry conditions, independently of the pumping rates, as long as pumping is retained above threshold. 

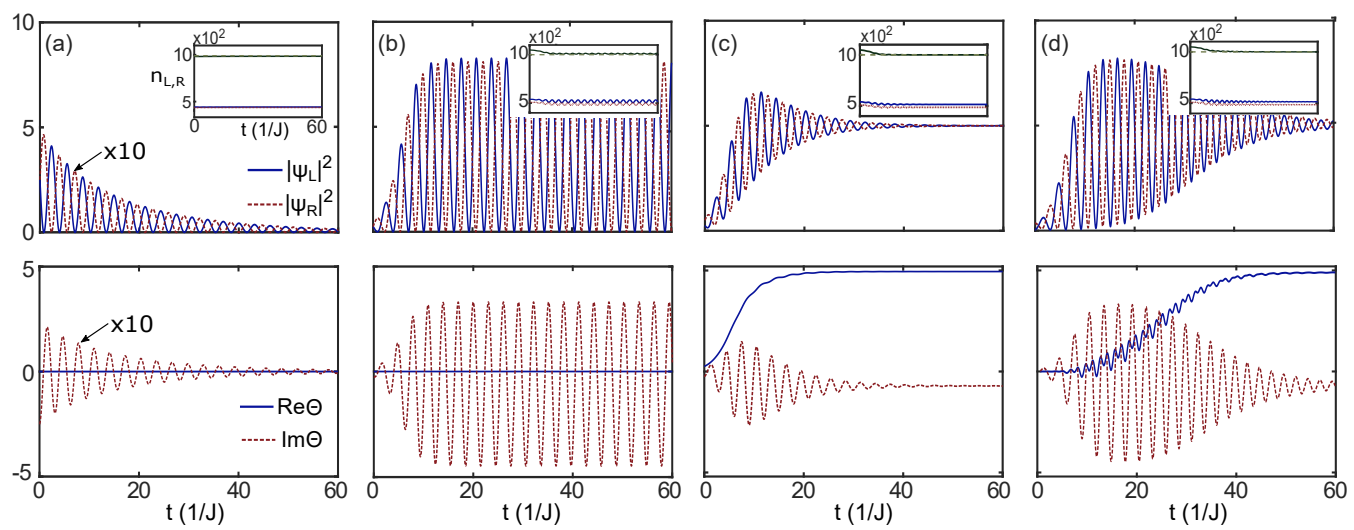

Figure 2. Dynamics of the polariton populations $\left|\psi_{L, R}\right|^{2}$ (upper panels), the reservoir excitons $n_{L, R}$ (insets), and the coherence $\Theta$ (lower panels), as obtained from the numerical solution of Equations (1) and (2) for the parameters $\epsilon_{L, R}=0, \kappa=10 \mathrm{~J}, \Gamma=2 \mathrm{~J}, R=0.02 \mathrm{~J}$ (time is in units of $J^{-1}$ ), corresponding to the threshold values of pumping $P_{L}+P_{R}=2 \kappa \Gamma / R=2000 J$ and the steadystate exciton populations $n_{L}+n_{R}=2 \kappa / R=1000$ (green dashed lines in the insets). The initial exciton populations are always taken as $n_{L, R}=P_{L, R} / \Gamma$, while the initial polariton amplitudes $\psi_{L, R}$ are seeded with small (complex) values. (a) Linear case $(\eta=0)$ with the pumping rates $P_{L}=1000 \mathrm{~J}$ and $P_{R}=990 \mathrm{~J}$ being slightly below threshold, leading to a decay in the initial polariton populations $\left|\psi_{L}(t)\right|^{2}$ and $\left|\psi_{R}(t)\right|$ and steady-state exciton population $n_{L}+n_{R}<2 \kappa / R$. (b) Same as in (a), but for stronger pumping rates $P_{L}=1080$ and $P_{R}=1020$ above threshold and initial conditions $\operatorname{Re} \Theta(0)=0$, leading to the initial build-up of the polariton populations and their continuous Rabi-like oscillations, while $\operatorname{Re} \Theta(t)=0 \forall t$, and the exciton population $n_{L}+n_{R}$ oscillating slightly above the threshold value $2 \kappa / R=1000$. (c) Same as in (b), but for the initial conditions $\operatorname{Re} \Theta(0) \neq 0$, leading to a steady-state of the system. (d) Same as in (b) with the initial conditions $\operatorname{Re} \Theta(0)=0$, but in the presence of nonlinearity $\eta=0.1 \mathrm{~J}$ that couples $\operatorname{Re} \Theta$ and $\operatorname{Im} \Theta$, leading to a steady-state of the system.

\section{Equivalence of the PT-Symmetry and Steady State Conditions}

To understand the dynamics of the system, it is convenient to express Equation (1) in terms of the polariton populations $\left|\psi_{L, R}\right|^{2}$ and coherence $\Theta \equiv \psi_{L} \psi_{R}^{*}$ as

$$
\begin{aligned}
\partial_{t}\left|\psi_{L}\right|^{2}= & 2 \gamma_{L}\left|\psi_{L}\right|^{2}+2 J \operatorname{Im} \Theta, \\
\partial_{t}\left|\psi_{R}\right|^{2}= & 2 \gamma_{R}\left|\psi_{R}\right|^{2}-2 J \operatorname{Im} \Theta, \\
\partial_{t} \Theta= & -i\left[\epsilon_{L}-\epsilon_{R}+\eta\left(\left|\psi_{L}\right|^{2}-\left|\psi_{R}\right|^{2}\right)\right] \Theta \\
& +\left(\gamma_{L}+\gamma_{R}\right) \Theta-i J\left(\left|\psi_{L}\right|^{2}-\left|\psi_{R}\right|^{2}\right) .
\end{aligned}
$$

Note that, below threshold, $\left(\gamma_{L}+\gamma_{R}\right)<0$, both the polariton populations and their coherence decay to zero, as mentioned above.

Let us set, without loss of generality, $\epsilon_{L, R}=0$ and consider first the case of vanishing nonlinearity $\eta=0$. Equation (7c) indicates that the coherence only decays if its real part is nonzero. In turn, the solution for the real part of the coherence is

$$
\operatorname{Re} \Theta(t)=\operatorname{Re} \Theta(0) e^{\int_{0}^{t}\left(\gamma_{L}+\gamma_{R}\right) d t^{\prime}}
$$

Hence, if initially $\operatorname{Re} \Theta(0)=0$, it will remain so at later times, $\operatorname{Re} \Theta(t)=0 \forall t>0$. Then, the dynamics of the system, if pumped above threshold, will exhibit continuous Rabi-like oscillations with frequency $J$, while no steady-state will be attained, as in Figure $2 b$.

In practice, however, even if we initially have $\operatorname{Re} \Theta(0)=0$ (e.g., either $\psi_{L}(0)=0$ or $\left.\psi_{R}(0)=0\right)$, the unavoidable phase fluctuations in the polaritons will eventually lead to the appearance of finite $\operatorname{Re} \Theta(t) \neq 0$, which, in turn, will result in the decay of coherence and drive the system to the steady state. Equivalently, if we initially have $\operatorname{Re} \Theta(0) \neq 0$, the system can still initially exhibit Rabi-like oscillations, but will eventually attain the 
steady state, as in Figure 2c. Finally, as seen from Equation (7c), the nonlinear interaction couples the real and imaginary parts of the coherence $\Theta$ with the rate $\eta\left(\left|\psi_{L}\right|^{2}-\left|\psi_{R}\right|^{2}\right)$. Hence, in the presence of nonlinearity $\eta \neq 0$, we expect the eventual decay of the coherence with the system attaining steady state, for any initial conditions and independent of the phase fluctuations, as in Figure 2d.

Setting the time derivative in the left-hand side of the Equation (7c) equal to zero, we find that the steady state is reached when

$$
R\left[n_{L}+n_{R}\right]-2 \kappa=0, \quad \text { and } \quad\left|\psi_{L}\right|^{2}=\left|\psi_{R}\right|^{2} .
$$

Remarkably, the first equation corresponds exactly to the PT-symmetry condition $n_{L}+n_{R}=\frac{2 \kappa}{R}$ discussed above. Moreover, this condition is satisfied even in the presence of nonlinear interaction $\eta \neq 0$, because the equal polariton populations, as per the second equation, lead to exactly the same energy shifts $\eta\left|\psi_{L, R}\right|^{2}$ of the polaritons in both wells. In other words, for any initial conditions, and provided the total pumping is above threshold as per Equation (6) but otherwise arbitrary $P_{L}$ and $P_{R}$, the system attains a stable fixed point corresponding to the PT-symmetric state. Even when no steady state exists or has yet been reached, the PT condition in Equation (9) is approximately satisfied, as seen in the insets of Figure 2.

Combining Equations (5) and (9), we find that steady state polariton populations are

$$
\left|\psi_{L}\right|^{2}=\left|\psi_{R}\right|^{2}=\frac{P_{L}+P_{R}}{2 \kappa}-\frac{\Gamma}{R}
$$

while the exciton populations are

$$
n_{L, R}=\frac{2 \kappa P_{L, R}}{R\left(P_{L}+P_{R}\right)}
$$

Using these stationary values for $n_{L, R}$ and $\left|\psi_{L, R}\right|^{2}$ in Equations (7a) and (7b) in the steady state, we obtain

$$
\operatorname{Im}(\Theta)=\frac{\kappa \Gamma\left(P_{L}-P_{R}\right)}{2 R J\left(P_{L}+P_{R}\right)}-\frac{P_{L}-P_{R}}{4 J},
$$

and from $\left|\psi_{L} \psi_{R}^{*}\right|^{2}=\left[\operatorname{Re}\left(\psi_{L} \psi_{R}^{*}\right)\right]^{2}+\left[\operatorname{Im}\left(\psi_{L} \psi_{R}^{*}\right)\right]^{2}$ we obtain

$$
\operatorname{Re}(\Theta)=\mathcal{D} \sqrt{4 J^{2}\left(P_{L}+P_{R}\right)^{2}-\kappa^{2}\left(P_{L}-P_{R}\right)^{2}}
$$

where

$$
\mathcal{D}=\frac{P_{L}+P_{R}-2 \kappa \Gamma / R}{4 J \gamma\left(P_{L}+P_{R}\right)} .
$$

These results are verified by the numerical simulations illustrated in Figure 2 and they equally hold for any value the nonlinearity strength $\eta$.

\section{Phase Fluctuations}

As mentioned above, the coherence of the polariton condensate will decay due to the phase fluctuations that are always present in realistic quantum systems. We, therefore, incorporate the phase noise in our numerical calculations and investigate how it modifies the dynamics of the polaritons and their coherence. We model the phase fluctuations as the standard Wiener process for stochastic differential equations. Thus, the single-particle energies $\epsilon_{L, R}$ in Equation (1) become Gaussian stochastic variables with the mean $\left\langle\epsilon_{L, R}\right\rangle=0$ and variance $\sigma^{2}=2 \xi / \delta t$, where $\xi$ is the decoherence rate and $\delta t$ is the time step used to select a new random energy.

In Figure 3 we show the results of our numerical simulations, as obtained upon the ensemble average over $N=1000$ independent realizations of the system dynamics. We 
compute the first-order correlation functions $g^{(1)}(t)$, which quantify the coherence for the polaritonic fields, via

$$
g^{(1)}(t)=\frac{\left\langle\psi\left(t_{0}\right) \psi(t)\right\rangle}{\sqrt{\left\langle\left|\psi\left(t_{0}\right)\right|^{2}\right\rangle\left\langle|\psi(t)|^{2}\right\rangle}},
$$

where $\psi=\psi_{L}$ or $\psi_{R}$, and $\langle\ldots\rangle$ denotes the ensemble average.
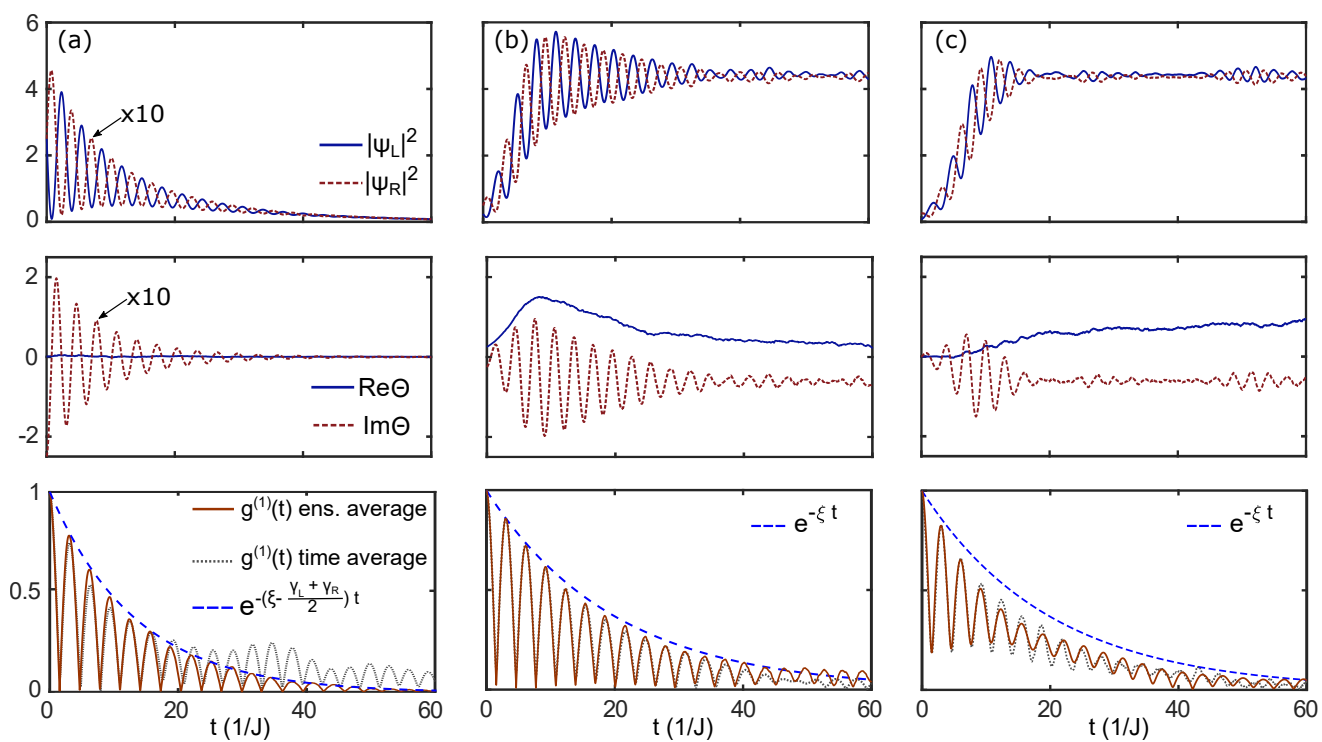

Figure 3. Dynamics of the polariton populations $\left|\psi_{L, R}\right|^{2}$ (upper panels), the coherence $\Theta$ (middle panels), and the left-well polariton field correlation function $g^{(1)}(t)$ (lower panels), for the same parameters as in Figure 2 with the addition of phase fluctuations causing decoherence with rate $\xi=0.05 \mathrm{~J}$, as obtained from the ensemble averaged solution of Equations (1) and (2). (a) Linear case $(\eta=0)$ with the below threshold pumping $P_{L}=1000 \mathrm{~J}$ and $P_{R}=990 \mathrm{~J}$ leading to the exponential decay in the correlation function $g^{(1)}(t) \propto e^{-\left(\xi-\left|\gamma_{L}+\gamma_{R}\right| / 2\right) t}$. (b) Same as in (a), but for stronger pumping above threshold $P_{L}=1080$ and $P_{R}=1020$; independently of the initial value of $\operatorname{Re} \Theta(0)$, the phase fluctuations cause an exponential decay in $g^{(1)}(t) \propto e^{-\xi t}$ while the system approaches the steady state. (c) Same as in (b) but in the presence of nonlinearity $\eta=0.1 \mathrm{~J}$, causing a slightly accelerated decay of $g^{(1)}(t) \propto e^{-\xi^{\prime} t}$ with $\xi^{\prime}>\xi$, and faster approach of the system to the steady state. In the lower panels, we also show the correlation functions $g^{(1)}(t)$ obtained from the long-term average of the system dynamics (the oscillating tail of $g^{(1)}$ is due to the finite length of the time series).

Below the pumping threshold, the polariton fields decay with rate $\gamma_{L}+\gamma_{R}<0$, but the phase fluctuations with rate $\xi$ causes an even faster decay of coherence, $g^{(1)}(t) \propto e^{-\left(\xi-\left|\gamma_{L}+\gamma_{R}\right| / 2\right) t}$, as seen in Figure 3a. For pumping above threshold, the phase noise causes an exponential decay in the correlation function $g^{(1)}(t) \propto e^{-\xi^{t}}$, while the system approaches the steady state independently of the initial value of coherence $\operatorname{Re} \Theta(0)$, as seen in Figure $3 b$. Including the nonlinear interaction $\eta \neq 0$ further accelerates the decay of the correlation function and the system approaches the steady state even faster, as seen in Figure 3c.

We finally note that, for an ergodic system, the ensemble-averaged and time-averaged correlation functions are equivalent. To verify whether our polariton system is ergodic, we also compute the field correlation function

$$
g^{(1)}(t)=\frac{\int_{t_{\mathrm{i}}}^{t_{\mathrm{f}}} d \tau \psi(\tau) \psi^{*}(\tau+t)}{\sqrt{\int_{t_{\mathrm{i}}}^{t_{\mathrm{f}}} d \tau|\psi(\tau)|^{2} \int_{t_{\mathrm{i}}}^{t_{\mathrm{f}}} d \tau|\psi(\tau+t)|^{2}}}
$$

resulting from a single, long-time trajectory with $t_{\mathrm{f}}-t_{\mathrm{i}}=3000 / \mathrm{J}$. As seen in Figure 3 (lower panels), the computed ensemble-averaged and time-averaged correlation functions coincide to a very approximation, attesting to the ergodicity of our system. 


\section{Conclusions}

In summary, we have studied an exciton-polariton system in a double-well potential, taking into account the dynamics of the reservoir excitons and the polaritons. We have found that for pumping of the excitons above the total threshold value to form the polariton condensate, the exciton populations attain values that satisfy the PT-symmetry condition for the polariton condensate, independent of the pumping rates of the individual wells. Employing the population-coherence equations, we interpreted the corresponding dynamics and revealed the stable fixed point, or the steady state, that the system approaches. To make our analysis experimentally relevant, we also included the phase fluctuations present in any realistic system, and computed the first-order correlation functions for the polariton fields, which revealed the coherence decay with the corresponding rate.

We note that our results apply to moderate non-linear interaction strength and small differences in pumping rates of the two wells. For large differences in the pumping rates, the strong non-linear energy shift in the polariton condensate energy may lead to the self-trapping and break-up of the PT symmetry [31].

Author Contributions: The authors contributed equally to this research and writing and editing the manuscript. Both authors have read and agreed to the published version of the manuscript.

Funding: This research was co-funded by Greece (General Secretariat for Research and Technology), and the European Union (European Regional Development Fund), in the framework of the bilateral Greek-Russian Science and Technology collaboration on Quantum Technologies (POLISIMULATOR project).

Institutional Review Board Statement: Not applicable.

Informed Consent Statement: Not applicable.

Data Availability Statement: Not applicable.

Acknowledgments: We thank P.G. Savvidis, H. Ohadi, and A.F. Tzortzakakis for fruitful discussions.

Conflicts of Interest: The authors declare no conflict of interest.

\section{Appendix A. Polariton Condensate in a PT-Symmetric Double Well}

Consider a polariton condensate in a double-well potential described by the coupledmode equations

$$
\begin{aligned}
& i \partial_{t} \psi_{L}=\left(\epsilon_{L}+i \gamma_{L}\right) \psi_{L}+\eta\left|\psi_{L}\right|^{2} \psi_{L}-J \psi_{R} \\
& i \partial_{t} \psi_{R}=\left(\epsilon_{R}+i \gamma_{R}\right) \psi_{R}+\eta\left|\psi_{R}\right|^{2} \psi_{R}-J^{*} \psi_{L}
\end{aligned}
$$

where $\epsilon_{L, R}$ are the single-particle energies, $\gamma_{L, R}$ are the incoherent loss $(\gamma<0)$ or gain $(\gamma>0)$ rates at each well, $\eta$ is the nonlinear interaction strength, and $J$ is the Josephson coupling between the wells.

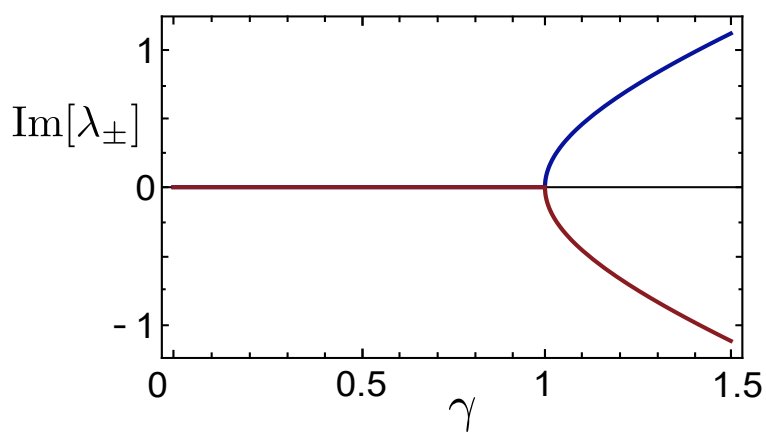

Figure A1. Imaginary part of the eigenvalues $\lambda_{ \pm}$in Equation (A3). For $\gamma<J(=1)$ we have a PT symmetric phase with real eigenvalues. Above the bifurcation point at $\gamma=J$, the system enters the PT broken phase with imaginary eigenvalues. 
Without loss of generality, we can set $\epsilon_{L, R}=0$. If we assume negligibly weak nonlinearity, $\eta|\psi|^{2} \ll J$, and set $\gamma_{L}=-\gamma_{R}=\gamma$ so that the loss at the right well is exactly compensated by the gain at the left well, we obtain a PT-symmetric Hamiltonian matrix corresponding to Equation (A1) [29]:

$$
\mathcal{H}=\left(\begin{array}{cc}
i \gamma & -J \\
-J^{*} & -i \gamma
\end{array}\right)
$$

Its eigenvalues and the corresponding eigenvectors are given by

$$
\lambda_{ \pm}= \pm \sqrt{|J|^{2}-\gamma^{2}}
$$

and

$$
| \pm\rangle=\left[\left(\sqrt{|J|^{2}-\gamma^{2}} \pm i \gamma\right)|L\rangle \mp J^{*}|R\rangle\right] / N_{ \pm}
$$

with $N_{ \pm}$the normalization factors. For $\gamma<|J|$, the eigenvalue spectrum is real and the dynamics is Hermitian-like. For $\gamma>|J|$, the eigenvalues become imaginary and the system enters the PT-broken phase. The case $|J|=\gamma$ corresponds to the exceptional point of the system where the eigenvalues become degenerate and the eigenstates coalesce. Figure A1 illustrates the dependence of imaginary part of the eigenvalues on the loss/gain parameter $\gamma$.

\section{References}

1. Deng, H.; Haug, H.; Yamamoto, Y. Exciton-polariton Bose-Einstein condensation. Rev. Mod. Phys. 2010, 82, 1489. [CrossRef]

2. Carusotto, I.; Ciuti, C. Quantum fluids of light. Rev. Mod. Phys. 2013, 85, 299. [CrossRef]

3. Lagoudakis, K.G.; Wouters, M.; Richard, M.; Baas, A.; Carusotto, I.; Andre, R.; Dang, L.S.; Deveaud-Pledran, B. Quantized vortices in an exciton-polariton condensate. Nat. Phys. 2008, 4, 706. [CrossRef]

4. Yamaguchi, M.; Kamide, K.; Nii, R.; Ogawa, T.; Yamamoto, Y. Second Thresholds in BEC-BCS-Laser Crossover of Exciton-Polariton Systems. Phys. Rev. Lett. 2013, 111, 026404. [CrossRef]

5. Schneider, C.; Rahimi-Iman, A.; Kim, N.Y.; Fischer, J.; Savenko, I.G.; Amthor, M.; Lermer, M.; Wolf, A.; Worschech, L.; Kulakovskii, V.D.; et al. An electrically pumped polariton laser. Nature 2013, 497, 348. [CrossRef]

6. Goblot, V.; Nguyen, H.S.; Carusotto, I.; Galopin, E.; Lematre, A.; Sagnes, I.; Amo, A.; Bloch, J. Phase-Controlled Bistability of a Dark Soliton Train in a Polariton Fluid. Phys. Rev. Lett. 2016, 117, 217401. [CrossRef] [PubMed]

7. Kasprzak, J.; Richard, M.; Kundermann, S.; Baas, A.; Jeambrun, P.; Keeling, J.; Marchetti, F.M.; Szymanska, M.H.; André, R.; Staehli, J.L.; et al. Bose-Einstein condensation of exciton polaritons. Nature 2006, 443, 409. [CrossRef]

8. Balili, R.; Hartwell, V.; Snoke, D.; Pfeiffer, L.; West, K. Bose-Einstein Condensation of Microcavity Polaritons in a Trap. Science 2007, 316, 1007. [CrossRef] [PubMed]

9. Amo, A.; Lefrére, J.; Pigeon, S.; Adrados, C.; Ciuti, C.; Carusotto, I.; Houdre, R.; Giacobino, E.; Bramati, A. Superfluidity of polaritons in semiconductor microcavities. Nat. Phys. 2009, 5, 805. [CrossRef]

10. Dominici, L.; Carretero-Gonzalez, R.; Cuevas-Maraver, J.; Gianfrate, A.; Rodrigues, A.S.; Frantzeskakis, D.J.; Kevrekidis, P.G.; Lerario, G.; Ballarini, D.; Giorgi, M.D.; et al. Interactions and scattering of quantum vortices in a polariton fluid. Nat. Comm. 2018, 9, 1467. [CrossRef]

11. Carretero-Gonzalez, R.; Cuevas-Maraver, J.; Frantzeskakis, D.J.; Horikis, T.P.; Kevrekidis, P.G.; Rodrigues, A.S. A Korteweg-de Vries description of dark solitons in polariton superfluids. Phys. Lett. A 2017, 381, 3805. [CrossRef]

12. Ohadi, H.; Ramsay, A.J;; Sigurdsson, H.; Redondo, Y.D.; Tsintzos, S.I.; Hatzopoulos, Z.; Liew, T.C.H.; Shelykh, I.A.; Rubo, Y.G.; Savvidis, P.G.; et al. Spin Order and Phase Transitions in Chains of Polariton Condensates. Phys. Rev. Lett. 2017, 119, 067401. [CrossRef]

13. Ohadi, H.; Redondo, Y.D.; Ramsay, A.J.; Hatzopoulos, Z.; Liew, T.C.H.; Eastham, P.R.; Savvidis, P.G.; Baumberg, J.J. Synchronization crossover of polariton condensates in weakly disordered lattices. Phys. Rev. B 2018, 97, 195109. [CrossRef]

14. Orfanakis, K.; Tzortzakakis, A.F.; Petrosyan, D.; Savvidis, P.G.; Ohadi, H. Ultralong temporal coherence in optically trapped exciton-polariton condensates. Phys. Rev. B 2021, 103, 235313. [CrossRef]

15. Harrison, S.L.; Sigurdsson, H.; Lagoudakis, P.G. Synchronization in optically trapped polariton Stuart-Landau networks. Phys. Rev. B 2020, 101, 155402. [CrossRef]

16. Töpfer, J.D.; Sigurdsson, H.; Alyatkin, S.; Lagoudakis, P.G. Lotka-Volterra population dynamics in coherent and tunable oscillators of trapped polariton condensates. Phys. Rev. B 2020, 102, 195428. [CrossRef]

17. Cherotchenko, E.D.; Sigurdsson, H.; Askitopoulos, A.; Nalitov, A.V. Optically controlled polariton condensate molecules. Phys. Rev. B 2021, 103, 115309. [CrossRef]

18. Zhang, X.; Zhang, Y.; Dong, H.; Tang, B.; Li, D.; Tian, C.; Xua, C.; Zhou, W. Room temperature exciton-polariton condensate in an optically-controlled trap Nanoscale 2019, 11, 4496. [CrossRef] 
19. Berloff, N.G.; Silva, M.; Kalinin, K.; Askitopoulos, A.; Töpfer, J.D.; Cilibrizzi, P.; Langbein, W.; Lagoudakis, P.G. Realizing the classical XY Hamiltonian in polariton simulators. Nat. Mat. 2017, 16, 1120. [CrossRef] [PubMed]

20. Su, R.; Ghosh, S.; Wang, J.; Liu, S.; Diederichs, C.; Liew, T.C.H.; Xiong, Q. Observation of exciton polariton condensation in a perovskite lattice at room temperature. Nat. Phys. 2020, 16, 301. [CrossRef]

21. Bender, C.M.; Brody, D.C.; Jones, H.F. Complex Extension of Quantum Mechanics. Phys. Rev. Lett. 2002, 89, 270401. [PubMed]

22. Bender, C.M.; Brody, D.C.; Jones, H.F. Extension of PT-symmetric quantum mechanics to quantum field theory with cubic interaction. Phys. Rev. D 2004, 70, 025001.

23. El-Ganainy, R.; Makris, K.G.; Christodoulides, D.N.; Musslimani, Z.H. Theory of coupled optical PT-symmetric structures. Opt. Lett. 2007, 32, 2632.

24. Fleury, R.; Sounas, D.; Alú, A. An invisible acoustic sensor based on parity-time symmetry. Nat. Commun. 2015, 6, 5905.

25. Chestnov, I.Y.; Demirchyan, S.S.; Alodjants, A.P.; Rubo, Y.G.; Kavokin, A.V. Permanent Rabi oscillations in coupled exciton-photon systems with PT-symmetry. Sci. Rep. 2016, 6, 19551.

26. Lien, J.-Y.; Chen, Y.-N.; Ishida, N.; Chen, H.-B.; Hwang, C.-C.; Nori, F. Multistability and condensation of exciton-polaritons below threshold. Phys. Rev. B 2015, 91, 024511.

27. Khurgin, J.B.Exceptional points in polaritonic cavities and subthreshold Fabry-Perot lasers. Optica 2020, 7, 1015.

28. Hanai, R.; Littlewood, P.B. Critical fluctuations at a many-body exceptional point. Phys. Rev. Res. 2020, 2, 033018.

29. Kalozoumis, P.A.; Nikolopoulos, G.M.; Petrosyan, D. Coherent population oscillations and an effective spin-exchange interaction in a PT-symmetric polariton mixture. EPL 2020, 129, 37003.

30. Wouters, M.; Carusotto, I. Excitations in a Nonequilibrium Bose-Einstein Condensate of Exciton Polaritons. Phys. Rev. Lett. 2007, 99, 140402. [PubMed]

31. Sukhorukov, A.A.; Xu, Z.; Kivshar, Y.S. Nonlinear suppression of time reversals in PT-symmetric optical couplers. Phys. Rev. A 2010, 82, 043818. [CrossRef] 